\title{
Bi-Hamiltonian structure of the Oriented Associativity Equation
}

\author{
Maxim V. Pavlov ${ }^{1}$, Raffaele F. Vitolo ${ }^{2}$ \\ ${ }^{1}$ Lebedev Physical Institute of Russian Academy of Sciences, \\ Moscow, Leninskij Prospekt, 53; \\ ${ }^{2}$ Department of Mathematics and Physics, \\ University of Salento, Lecce, Italy \\ and INFN, Section of Lecce \\ http://poincare.unisalento.it/vitolo
}

\begin{abstract}
The Oriented Associativity equation plays a fundamental role in the theory of Integrable Systems. In this paper we prove that the equation, besides being Hamiltonian with respect to a first-order Hamiltonian operator, has a third-order non-local homogeneous Hamiltonian operator belonging to a class which has been recently studied, thus providing a highly non-trivial example in that class and showing intriguing connections with algebraic geometry.
\end{abstract}

MSC2010: 37K05.

\section{Introduction}

The Associativity equation, or Witten-Dijkgraaf-Verlinde-Verlinde (WDVV) equation, plays a fundamental role in the geometric theory of Integrable Systems. Its solutions define Frobenius manifolds, which correspond to integrable systems; Frobenius manifolds also play a fundamental role in the theory of quantum cohomology and Gromov-Witten invariants. These connections were shown by B. Dubrovin in his seminal paper [3]. 
The nonlinear partial differential system of equations

$$
\frac{\partial^{2} c^{i}}{\partial a^{j} \partial a^{m}} \frac{\partial^{2} c^{m}}{\partial a^{k} \partial a^{n}}=\frac{\partial^{2} c^{i}}{\partial a^{k} \partial a^{m}} \frac{\partial^{2} c^{m}}{\partial a^{j} \partial a^{n}}
$$

on $N$ unknown functions $\left(c^{i}\right)$ of $N$ independent variables $\left(a^{j}\right)$ was introduced in [13] as a generalization of the Associativity equations. Its solution define $F$-manifolds, which are still in correspondence with integrable systems. The far-reaching implication of this generalization are an active subject of study: flat and bi-flat $F$-manifolds have interesting connections with Painlevé equations $[1,18,19]$; see also the papers $[15,16])$ devoted to coisotropic deformations. We call the system (1) the Oriented Associativity equation.

The Oriented Associativity equation admits the scalar linear spectral problem

$$
\frac{\partial^{2} h}{\partial a^{i} \partial a^{j}}=\lambda \frac{\partial^{2} c^{m}}{\partial a^{i} \partial a^{j}} \frac{\partial h}{\partial a^{m}}
$$

(see, for instance, [3, 22])that ensure that the equation is integrable as it provides a Lax pair.

We observe that the Associativity equation [3] can be obtained from (1) by the potential reduction $c^{i}=\eta^{i m} \partial F / \partial a^{m}$, where $\eta^{k s}$ is a constant nondegenerate symmetric matrix.

In this paper, we will prove the existence of a bi-Hamiltonian formalism for the Oriented Associativity equation (1).

The above result has strong analogies with the known results on the Associativity (WDVV) equation. Indeed, the Associativity equation can be written as $N-2$ commuting hydrodynamic-type systems of conservation laws [9]. For $N=3$ the (only) system was shown to be bi-Hamiltonian in [8]. Further investigations shown a similar situation in the case $N=4$ : the Hamiltonian operators were, in both cases, a first-order homogeneous operator (found in [9] for $N=4$ ) and a third-order homogeneous operator (found in [23] for $N=4$ ). First-order homogeneous operators [4] can be written as $A_{1}^{i j}=g^{i j} \partial_{x}$, where $\left(g^{i j}\right)$ is a constant matrix, in a suitable coordinate system; third-order homogeneous operators [5] have a more complicated structure, and can be brought to the form

$$
A_{2}^{i j}=\partial_{x}\left(g^{i j} \partial_{x}+c_{k}^{i j} u_{x}^{k}\right) \partial_{x}
$$

Such operators have been extensively studied quite recently [10, 11]. 
Recently, a first-order homogeneous Hamiltonian operator for the simplest case $(N=3)$ of Oriented Associativity equation (written in the form of a hydrodynamic-type system) was found [22] with the same method as in the Associativity case. It was natural to conjecture that a third-order homogeneous Hamiltonian operator might exist.

In this paper, we will prove that the Oriented Associativity equation in hydrodynamic-type form admits a non-local third-order homogeneous Hamiltonian operator of a class that was recently introduced by M. Casati, E.V. Ferapontov and the authors of this paper in [2].

The significance of the result is high: indeed, it is known that the Associativity equations (in hydrodynamic form) in the cases $N=3$ and $N=4$ discussed above correspond to linear line congruences, which are algebraic varieties in the Plücker variety all lines of a projective space [12]. Their third-order Hamiltonian operators correspond to quadratic line complexes, which are algebraic varieties of lines in a projective space of different dimension with respect to the previous lines $[10,11]$.

The Oriented Associativity equation (in hydrodynamic form) can also be interpreted as a line congruence, even if we still do not know if the congruence is linear. The third-order non-local homogeneous Hamiltonian operator that we find as the main result in this paper also defines a quadratic line complex. It is thus clear that the strong links between the Associativity equation and projective-geometric varieties are preserved for the Oriented Associativity equation. We believe that such structures play an important role in the rich geometry of such equations, with lots of interesting Mathematics yet to be discovered.

The computation related to finding the non-local Hamiltonian operator is highly non-trivial, and it is made possible by a systematic use of computer algebra systems, in particular Reduce and its package CDE for computations with Hamiltonian operators [17, 24].

\section{The Oriented Associativity equation}

The system of quadratic equations

$$
\begin{aligned}
& u_{x x}=v_{x t} w_{x x}-v_{x x} w_{x t}+w_{x t}^{2}-w_{x x} w_{t t}, \\
& u_{x t}=v_{t t} w_{x x}-v_{x t} w_{x t} \\
& u_{t t}=v_{x t}^{2}-v_{x x} v_{t t}+v_{t t} w_{x t}-v_{x t} w_{t t}
\end{aligned}
$$


is the Oriented Associativity equation in the simplest case $N=3$. It is endowed by the Lax pair

$$
\begin{aligned}
& \left(\begin{array}{l}
\psi \\
\psi_{1} \\
\psi_{2}
\end{array}\right)_{x}=\lambda\left(\begin{array}{ccc}
0 & 1 & 0 \\
u_{x x} & v_{x x} & w_{x x} \\
u_{x t} & v_{x t} & w_{x t}
\end{array}\right)\left(\begin{array}{c}
\psi \\
\psi_{1} \\
\psi_{2}
\end{array}\right), \\
& \left(\begin{array}{c}
\psi \\
\psi_{1} \\
\psi_{2}
\end{array}\right)_{t}=\lambda\left(\begin{array}{ccc}
0 & 0 & 1 \\
u_{x t} & v_{x t} & w_{x t} \\
u_{t t} & v_{t t} & w_{t t}
\end{array}\right)\left(\begin{array}{c}
\psi \\
\psi_{1} \\
\psi_{2}
\end{array}\right)
\end{aligned}
$$

Let us introduce a new set of field variables $q^{1}=u_{x x}, q^{2}=u_{x t}, q^{3}=v_{x x}, q^{4}=$ $v_{x t}, q^{5}=w_{x x}, q^{6}=w_{x t}$. Then, the quadratic system (4) becomes the six component hydrodynamic type system of conservation laws

$$
\begin{aligned}
q_{t}^{1}=q_{x}^{2}, & q_{t}^{2}=\partial_{x} \frac{q^{2} q^{6}+q^{1} q^{4}-q^{2} q^{3}}{q^{5}}, \\
q_{t}^{3}=q_{x}^{4}, & q_{t}^{4}=\partial_{x} \frac{q^{2}+q^{4} q^{6}}{q^{5}}, \\
q_{t}^{5}=q_{x}^{6}, & q_{t}^{6}=\partial_{x} \frac{\left(q^{6}\right)^{2}-q^{3} q^{6}+q^{4} q^{5}-q^{1}}{q^{5}} .
\end{aligned}
$$

The above system (7) possesses at least two extra local conservation laws. One can prove that this system has also three extra conservation laws

$$
\partial_{t} v^{k}=\partial_{x} \frac{\left(v^{k}\right)^{2}-q^{3} v^{k}-q^{1}}{q^{5}}, \quad k=1,2,3,
$$

where $v^{i}$ are the roots of the characteristic polynomial $\lambda^{3}-\left(q^{3}+q^{6}\right) \lambda^{2}+$ $\left(q^{3} q^{6}-q^{4} q^{5}-q^{1}\right) \lambda+q^{1} q^{6}-q^{2} q^{5}$ of one of the matrices of the Lax pair (5). By Viète's theorem we have $q^{3}+q^{6}=v^{1}+v^{2}+v^{3}$, so that only two of the densities $v^{i}$ are new.

\section{$3 \quad$ First-order Hamiltonian structure}

The hydrodynamic-type system (7) admits a first-order homogeneous Hamiltonian operator. This class of Hamiltonian operators was first introduced in [4]. Operators in this class always admit a coordinate system in which they 
can be presented as $A_{1}=g^{i j} \partial_{x}$, where $g^{i j}$ is a constant matrix. The results in this sections were found in [22], using techniques that are analogous to those used in [8].

We can change the coordinates in the above hydrodynamic-type system to the new coordinates $\left(u^{k}\right)$ defined by the Viète formulae:

$$
\begin{array}{ll}
u^{1}=v^{1}, & u^{2}=v^{2}, \\
u^{3}=v^{3}, & u^{4}=q^{4}, \\
u^{5}=q^{5}, & u^{6}=2 q^{3}-\left(v^{1}+v^{2}+v^{3}\right) .
\end{array}
$$

They are related with $\left(q^{i}\right)$ by the formulae

$$
\begin{aligned}
q^{1} & =\frac{1}{4}\left(u^{1}+u^{2}+u^{3}\right)^{2}-\left(u^{1} u^{2}+u^{1} u^{3}+u^{2} u^{3}\right)-\frac{1}{4}\left(u^{6}\right)^{2}-u^{4} u^{5}, \\
q^{2} & =\frac{2 u^{1} u^{2} u^{3}+\left(u^{1}+u^{2}+u^{3}-u^{6}\right) q^{1}}{2 u^{5}}, \\
q^{3} & =\frac{1}{2}\left(u^{1}+u^{2}+u^{3}+u^{6}\right), \\
q^{4} & =u^{4} \\
q^{5} & =u^{5} \\
q^{6} & =\frac{1}{2}\left(u^{1}+u^{2}+u^{3}-u^{6}\right) .
\end{aligned}
$$

Note that the inverse formulae contain cubic roots, and have a much more complicated expression. In the coordinates $\left(u^{i}\right)$ a Hamiltonian formulation of the system becomes immediate:

$$
u_{t}^{i}=g^{i k} \partial_{x} \frac{\partial H}{\partial u^{k}}, \quad \text { where } \quad g^{i k}=-\left(\begin{array}{cccccc}
0 & 1 & 1 & 0 & 0 & 0 \\
1 & 0 & 1 & 0 & 0 & 0 \\
1 & 1 & 0 & 0 & 0 & 0 \\
0 & 0 & 0 & 0 & 1 & 0 \\
0 & 0 & 0 & 1 & 0 & 0 \\
0 & 0 & 0 & 0 & 0 & 2
\end{array}\right)
$$

the Hamiltonian density is $H=q^{2}$ and the momentum density is $P=q^{1}=$ $\frac{1}{2} \tilde{g}_{i k} u^{i} u^{k}$. 


\section{Third-order nonlocal operators and systems of conservation laws}

After the results in [22], we might be tempted to conjecture that, by analogy with the Associativity equation, also the Oriented Associativity equation is endowed by two local homogeneous Hamiltonian operators, of first order and third order. Strictly speaking, this is not true. We recall that the conditions for an operator of the form (3) to be Hamiltonian (provided $\operatorname{det}\left(g^{i j}\right) \neq 0$ ) are

$$
\begin{aligned}
& g_{i j}=g_{j i}, \\
& c_{n k m}=\frac{1}{3}\left(g_{m n, k}-g_{k n, m}\right) \\
& g_{i j, k}+g_{j k, i}+g_{k i, j}=0, \\
& c_{n m l, k}+c_{m l}^{s} c_{s n k}=0 .
\end{aligned}
$$

where $\left(g_{i j}\right)^{-1}=\left(g^{i j}\right)$ and $c_{i j k}=g_{i q} g_{j p} c_{k}^{p q}$. By repeating the procedure that led to the results in [23], we found a candidate $\left(g^{i j}\right)$ for a leading term of a third order homogeneous Hamiltonian operator. But that fulfills (12a), (12b) and $(12 \mathrm{c})$ but does not fulfill (12d). After the results in [2], we conjectured

that the third-order homogeneous Hamiltonian operator $B=\left(B^{i j}\right)$ might be nonlocal, of the type

$$
B^{i j}=\partial_{x} \circ F^{i j} \circ \partial_{x}=\partial_{x}\left(g^{i j} \partial_{x}+c_{k}^{i j} u_{x}^{k}+c^{\alpha} w_{\alpha k}^{i} u_{x}^{k} \partial_{x}^{-1} w_{\alpha h}^{j} u_{x}^{h}\right) \partial_{x}
$$

and $w_{\alpha k}^{i}=w_{\alpha k}^{i}\left(u^{j}\right)$, with $c^{\alpha} \in \mathbb{R}$. In such an ansatz $F^{i j}$ has the same structure as Ferapontov-Mokhov nonlocal first-order homogeneous operators $[21,6,7]$. However, the two compositions with $\partial_{x}$ change the conditions for the operator to be Hamiltonian to [2]

$$
\begin{aligned}
& w_{\alpha i j}+w_{\alpha j i}=0, \\
& w_{\alpha i j, l}-c_{i j}^{s} w_{\alpha s l}=0, \\
& c_{n m l, k}+c_{m l}^{s} c_{s n k}+c^{\alpha} w_{\alpha m l} w_{\alpha n k}=0,
\end{aligned}
$$

in addition to $(12 \mathrm{a}),(12 \mathrm{~b}),(12 \mathrm{c})$ (of course, (14c) is a modification of $(12 \mathrm{~d})$ ), where $w_{i j}=g_{i s} w_{j}^{s}$. We remain with the problem of determining the tensors $w_{\alpha j}^{i}$. It is known that in Ferapontov-Mokhov case they are matrices of commuting flows with respect to the hydrodynamic-type system of which the 
operator is Hamiltonian. In this case, that is false: the condition of compatibility between $B$ and the Oriented Associativity equation (7) can be derived by the condition that the Hamiltonian operator maps conserved quantities into symmetries. It was shown in [14] that such a condition is equivalent to finding solutions $B$ to the equation

$$
\ell_{E}(B(\mathbf{p}))=0,
$$

over the adjoint system (or cotangent covering)

$$
\left\{\begin{array}{l}
E=0 \\
\ell_{E}^{*}(\mathbf{p})=0,
\end{array}\right.
$$

where $\mathbf{p}$ is an auxiliary (vector) variable, $E^{i}=u_{t}^{i}-\left(V^{i}\right)_{x}=0$ is the initial equation, with $V^{i}=V^{i}(\mathbf{u})$ the vector of fluxes, and $\ell_{E}$ is the formal linearization (Fréchet derivative) of $E$ and $\ell_{E}^{*}$ its adjoint operator.

It is easier to compute the condition (15) in potential coordinates $b_{x}^{i}=u^{i}$. We have

$$
B^{i j}=-\left(g^{i j}\left(\mathbf{b}_{x}\right) \partial_{x}+c_{k}^{i j}\left(\mathbf{b}_{x}\right) b_{x x}^{k}+c^{\alpha} w_{\alpha k}^{i}\left(\mathbf{b}_{x}\right) b_{x x}^{k} \partial_{x}^{-1} w_{\alpha h}^{j}\left(\mathbf{b}_{x}\right) b_{x x}^{h}\right)
$$

and $E^{i}=b_{t}^{i}-V^{i}\left(\mathbf{b}_{x}\right)$. Let us introduce the notation

$$
\frac{\partial V^{i}}{\partial b_{x}^{k}}=V_{k}^{i}, \quad \frac{\partial V^{i}}{\partial b_{x}^{k} \partial b_{x}^{h}}=V_{k h}^{i}, \quad g_{, k}^{i j}=\frac{\partial g^{i j}}{\partial b_{x}^{k}}, \quad c_{k, h}^{i j}=\frac{\partial c_{k}^{i j}}{\partial b_{x}^{h}},
$$

and similarly for other derivatives. We have

$$
\ell_{F}(\boldsymbol{\varphi})=\partial_{t} \varphi^{i}-V_{j}^{i} \partial_{x} \varphi^{j}, \quad \ell_{F}^{*}(\boldsymbol{\psi})=-\partial_{t} \psi_{k}+\partial_{x}\left(V_{k}^{i} \psi_{i}\right) .
$$

so that the adjoint system is

$$
\begin{aligned}
& b_{t}^{i}=V^{i}\left(\mathbf{b}_{x}\right) \\
& p_{k, t}=V_{k h}^{i} b_{x x}^{h} p_{i}+V_{k}^{i} p_{i, x}
\end{aligned}
$$

If we assume that $w_{\alpha j}^{i}\left(\mathbf{b}_{x}\right) b_{x x}^{j}$ are hydrodynamic-type symmetries of the system (19) then we can prove that they yield conservation laws on the adjoint system whose densities and fluxes are, respectively:

$$
r_{\alpha t}=V_{j}^{i} w_{\alpha k}^{j} b_{x x}^{k} p_{i}, \quad r_{\alpha x}=w_{\alpha k}^{i} b_{x x}^{k} p_{i} .
$$

The potential variables $r_{\alpha}$ allow us to represent the operator as

$$
B^{i}(\mathbf{p})=-g^{i j} p_{j, x}-c_{k}^{i j} b_{x x}^{k} p_{j}-c^{\alpha} w_{\alpha k}^{i} b_{x x}^{k} r_{\alpha} .
$$


Lemma 1 The condition $\ell_{F}(B(\mathbf{p}))=0$ is equivalent to the conditions:

$$
\begin{aligned}
& -g^{i j} V_{j}^{h}+V_{j}^{i} g^{j h}=0 \\
& -g_{, k}^{i h} V_{l}^{k}-g^{i j} 2 V_{j l}^{h}-c_{l}^{i j} V_{j}^{h}+V_{j}^{i} g_{, l}^{j h}+V_{j}^{i} c_{l}^{j h}=0 \\
& -g^{i k} V_{k l}^{h}-c_{k}^{i h} V_{l}^{k}+V_{k}^{i} c_{l}^{k h}=0 . \\
& -g^{i j} V_{j l m}^{h}-\frac{1}{2}\left(c_{m, j}^{i h} V_{l}^{j}+c_{l, j}^{i h} V_{m}^{j}\right)-c_{k}^{i h} V_{l m}^{k} \\
& \quad-\frac{1}{2}\left(c_{m}^{i j} V_{j l}^{h}+c_{l}^{i j} V_{j m}^{h}\right)+\frac{1}{2}\left(V_{j}^{i} c_{m, l}^{j h}+V_{j}^{i} c_{l, m}^{j h}\right) \\
& \quad-c^{\alpha} \frac{1}{2}\left(V_{k}^{h}\left(w_{\alpha l}^{i} w_{\alpha m}^{k}+w_{\alpha m}^{i} w_{\alpha l}^{k}\right)+V_{j}^{i}\left(w_{\alpha l}^{j} w_{\alpha m}^{h}+w_{\alpha m}^{j} w_{\alpha l}^{h}\right)\right)=0 \\
& -w_{\alpha h, k}^{i} V_{m}^{k}-w_{\alpha m, k}^{i} V_{h}^{k}-w_{\alpha k}^{i} V_{m, h}^{k} \\
& \quad-w_{\alpha k}^{i} V_{h, m}^{k}+V_{k}^{i} w_{\alpha m, h}^{k}+V_{k}^{i} w_{\alpha h, m}^{k}=0 \\
& -w_{\alpha k}^{i} V_{h}^{k}+V_{k}^{i} w_{\alpha h}^{k}=0
\end{aligned}
$$

Proof. We have:

$$
\begin{aligned}
\ell_{F}( & B(\mathbf{p}))^{i}= \\
= & -g_{, k}^{i j} b_{x t}^{k} p_{j, x}-g^{i j} p_{j, x t}-c_{k, h}^{i j} b_{x t}^{h} b_{x x}^{k} p_{j}-c_{k}^{i j} b_{x x t}^{k} p_{j}-c_{k}^{i j} b_{x x}^{k} p_{j, t} \\
& +V_{j}^{i}\left(g_{, h}^{j k} b_{x x}^{h} p_{k, x}+g^{j k} p_{k, x x}+c_{k, l}^{j h} b_{x x}^{l} b_{x x}^{k} p_{h}+c_{k}^{j h} b_{x x x}^{k} p_{h}+c_{k}^{j h} b_{x x}^{k} p_{h, x}\right) \\
& -c^{\alpha} w_{\alpha k, h}^{i} b_{x t}^{h} b_{x x}^{k} r_{\alpha}-c^{\alpha} w_{\alpha k}^{i} b_{x x t}^{k} r_{\alpha}-c^{\alpha} w_{\alpha k}^{i} b_{x x}^{k} V_{l}^{h} w_{\alpha m}^{l} b_{x x}^{m} p_{h} \\
& +V_{j}^{i} c^{\alpha}\left(w_{\alpha k, h}^{j} b_{x x}^{h} b_{x x}^{k} r_{\alpha}+w_{\alpha k}^{j} b_{x x x}^{k} r_{\alpha}+w_{\alpha k}^{j} b_{x x}^{k} w_{\alpha l}^{h} b_{x x}^{l} p_{h}\right) .
\end{aligned}
$$

After replacing the derivatives $b_{x t}^{h}, b_{x x t}^{h}$ and $p_{j, x t}$ using the equations (19) and (20) we obtain a polynomial in $p_{j}, b_{x x}^{k}$ and higher $x$-derivatives; its coefficient shall vanish, they are are the conditions of the statement.

Remark 2 A direct computation shows that the two flows $V^{i}$ and $w_{\alpha k}^{j} u_{x x}^{k}$ commute if and only if the conditions (23e) and (23f) hold true. Moreover, by arguments that are similar to those of [12, Theorem 1] it can be proved that (23d) is a consequence of the other equations (23) and (12), (14).

\section{Third-order Hamiltonian structure}

It is known[10] that $g_{i j}$ shall be a polynomial of second degree. Then, equations $(23 \mathrm{a}),(23 \mathrm{~b}),(23 \mathrm{c})$ are easily solved with respect to $\left(g_{i j}\right)$, where $V^{i}$ is 
the vector of fluxes of the Oriented Associativity equation (7). We obtain the unique solution:

$$
\begin{aligned}
& \left(g_{i j}\right)= \\
& \left(\begin{array}{cccc}
2 & 0 & q^{3} & -q^{5} \\
0 & 0 & 2 q^{5} & 0 \\
q^{3} & 2 q^{5} & -2\left(q^{1}+q^{4} q^{5}\right) & q^{5}\left(q^{3}-q^{6}\right) \\
-q^{5} & 0 & q^{5}\left(q^{3}-q^{6}\right) & 2\left(q^{5}\right)^{2} \\
2 q^{4} & -q^{3}+2 q^{6} & -q^{2}+q^{4}\left(q^{3}-q^{6}\right) & -q^{1}+q^{3}\left(-q^{3}+2 q^{6}\right)-2 q^{4} q^{5}-\left(q^{6}\right)^{2} \\
0 & -q^{5} & 2 q^{4} q^{5} & q^{5}\left(-q^{3}+q^{6}\right)
\end{array}\right. \\
& \left.\begin{array}{cc}
2 q^{4} & 0 \\
-q^{3}+2 q^{6} & -q^{5} \\
-q^{2}+q^{4}\left(q^{3}-q^{6}\right) & 2 q^{4} q^{5} \\
-q^{1}+q^{3}\left(-q^{3}+2 q^{6}\right)-2 q^{4} q^{5}-\left(q^{6}\right)^{2} & q^{5}\left(-q^{3}+q^{6}\right) \\
2\left(q^{4}\right)^{2} & -q^{2}+q^{4}\left(-q^{3}+q^{6}\right) \\
-q^{2}+q^{4}\left(-q^{3}+q^{6}\right) & -2 q^{4} q^{5}
\end{array}\right)
\end{aligned}
$$

We stress that $\left(g_{i j}\right)$ does not fulfill $(12 \mathrm{~d})$. Hence, we shall compute suitable tensors $w_{\alpha j}^{i}$.

A direct computation of $w_{\alpha j}^{i}$ as symmetries of (19) is very heavy. Since we have at our disposal a Lax pair, we can compute a sequence of homogeneous conserved quantities with a standard technique in the theory of integrable systems; see [23] for details. Then, we can transform them into symmetries using the Hamiltonian operator. We rewrite (5) in terms of $\left(q^{i}\right)$ and get

$$
\left(\begin{array}{c}
\psi \\
\psi^{1} \\
\psi^{2}
\end{array}\right)_{x}=\lambda\left(\begin{array}{ccc}
0 & 1 & 0 \\
q^{1} & q^{3} & q^{5} \\
q^{2} & q^{4} & q^{6}
\end{array}\right)\left(\begin{array}{c}
\psi \\
\psi^{1} \\
\psi^{2}
\end{array}\right) .
$$

By eliminating $\psi^{1}, \psi^{2}$ from (25) we obtain the single linear PDE

$$
\begin{aligned}
& \left(-q_{x}^{1} \lambda^{2} q^{5}+q_{x}^{5} \lambda^{2} q^{1}+\lambda^{3} q^{1} q^{5} q^{6}-\lambda^{3} q^{2}\left(q^{5}\right)^{2}\right) \psi+ \\
& \left(-q_{x}^{3} \lambda q^{5}+q_{x}^{5} \lambda q^{3}-\lambda^{2} q^{1} q^{5}+\lambda^{2} q^{3} q^{5} q^{6}-\lambda^{2} q^{4}\left(q^{5}\right)^{2}\right) \psi_{x} \\
& \quad+\left(-q_{x}^{5}-\lambda q^{3} q^{5}-\lambda q^{5} q^{6}\right) \psi_{2 x}+q^{5} \psi_{3 x}=0
\end{aligned}
$$

The substitution $\psi=\exp \int r d x$ yields a nonlinear ordinary differential equation on the function $r$ and its first, second and third order derivatives. This function $r$ plays the role of a generating function of conservation law densities with respect to the parameter $\lambda$ for the system (7). The expansion of $r$ at infinity (i.e. $\lambda \rightarrow \infty$ )

$$
r=\lambda h_{-1}+h_{0}+\frac{h_{1}}{\lambda}+\frac{h_{2}}{\lambda^{2}}+\ldots
$$


in the above equation leads to a sequence of differential relationships between the coefficients $h_{-1}, h_{0}, h_{1}, \ldots$ The leading term (the coefficient of $\lambda^{3}$ ) coincides with the characteristic equation of the eigenvalues of the matrix in (25). Thus, starting from $h_{-1}=u^{k}, k=1,2,3$, the expansion of $r$ with respect to $\lambda$ has three branches of conservation law densities, that we denote by $h_{i k}, i=0,1, \ldots$ Such densities are quasihomogeneous polynomials of degrees $\operatorname{deg} h_{i k}=i+1$ with respect to the grading $\operatorname{deg} u=0, \operatorname{deg} \partial_{x}=1$, and their coefficients are expressible via rational functions of $\left(u^{k}\right)$.

Using Reduce [17] we found all expressions of $h_{i k}$, for $k=1,2,3$ and $i=0$ : they are of the form $h_{0 k}=c_{k i}(u) u_{x}^{i}$, where

$$
\begin{gathered}
h_{01}=\frac{1}{S_{1}}\left(-4 u_{x}^{4}\left(u^{5}\right)^{2}+u_{x}^{5}\left(\left(u^{6}\right)^{2}-2 u^{6} u^{1}+\left(u^{1}\right)^{2}-\left(u^{2}\right)^{2}\right.\right. \\
\left.+2 u^{2} u^{3}-\left(u^{3}\right)^{2}\right)+2 u_{x}^{6} u^{5}\left(-u^{6}+u^{1}\right)+2 u_{x}^{1} u^{5}\left(-2 u^{1}+u^{2}+u^{3}\right) \\
\left.+2 u_{x}^{2} u^{5}\left(u^{2}-u^{3}\right)+2 u_{x}^{3} u^{5}\left(-u^{2}+u^{3}\right)\right) \\
h_{02}=\frac{1}{S_{2}}\left(4 u_{x}^{4}\left(u^{5}\right)^{2}+u_{x}^{5}\left(-\left(u^{6}\right)^{2}+2 u^{6} u^{2}+\left(u^{1}\right)^{2}-2 u^{1} u^{3}\right.\right. \\
\left.-\left(u^{2}\right)^{2}+\left(u^{3}\right)^{2}\right)+2 u_{x}^{6} u^{5}\left(u^{6}-u^{2}\right)+2 u_{x}^{1} u^{5}\left(-u^{1}+u^{3}\right)+ \\
\left.2 u_{x}^{2} u^{5}\left(-u^{1}+2 u^{2}-u^{3}\right)+2 u_{x}^{3} u^{5}\left(u^{1}-u^{3}\right)\right) \\
h_{03}=\frac{1}{S_{3}}\left(-4 u_{x}^{4}\left(u^{5}\right)^{2}+u_{x}^{5}\left(\left(u^{6}\right)^{2}-2 u^{6} u^{3}-\left(u^{1}\right)^{2}+2 u^{1} u^{2}\right.\right. \\
\left.-\left(u^{2}\right)^{2}+\left(u^{3}\right)^{2}\right)+2 u_{x}^{6} u^{5}\left(-u^{6}+u^{3}\right)+2 u_{x}^{1} u^{5}\left(u^{1}-u_{2}\right) \\
\left.+2 u_{x}^{2} u^{5}\left(-u^{1}+u^{2}\right)+2 u_{x}^{3} u^{5}\left(u^{1}+u^{2}-2 u^{3}\right)\right)
\end{gathered}
$$

where $S_{1}=4 u^{5}\left(\left(u^{1}\right)^{2}-u^{1} u^{2}-u^{1} u^{3}+u^{2} u^{3}\right), S_{2}=4 u^{5}\left(u^{1} u^{2}-u^{1} u^{3}-\left(u^{2}\right)^{2}+\right.$ $\left.u^{2} u^{3}\right) S_{3}=4 u^{5}\left(u^{1} u^{2}-u^{1} u^{3}-u^{2} u^{3}+\left(u^{3}\right)^{2}\right)$.

We observe that the above conserved densities are not independent. It holds:

$$
h_{01}+h_{02}+h_{03}=0 .
$$

We get (higher) commuting flows from the above conserved densities by the formula in potential coordinates

$$
b_{t}^{i}=\tilde{g}^{i k} \partial_{x}^{-1} \frac{\delta h}{\delta b^{k}}=\tilde{g}^{i k}\left(-\frac{\partial h}{\partial b_{x}^{k}}+\partial_{x} \frac{\partial h}{\partial b_{x x}^{k}}\right)=w_{j}^{i}\left(\mathbf{b}_{x}\right) b_{x x}^{j} .
$$


In particular, from the independent densities $h_{01}$ and $h_{02}$ we obtain the commuting flows $w_{1 j}^{i}\left(\mathbf{b}_{x}\right) b_{x x}^{j}$ and $w_{2 j}^{i}\left(\mathbf{b}_{x}\right) b_{x x}^{j}$. We observe that they are commuting flows for the system (19) in potential coordinates, and they define higher commuting flows $\left(w_{\alpha j}^{i} u_{x}^{j}\right)_{x}$ for the system (9). So, they fulfill the conditions (23e) and (23f) as they are invariant conditions. However, we need to check the Hamiltonian property of the operator $B$ in coordinates $\left(q^{i}\right)$.

In principle, it is possible to invert the coordinate change (10) and express the commuting flows $\left(w_{\alpha j}^{i} u_{x}^{j}\right)_{x}$ in coordinates $\left(q^{i}\right)$.

Lemma 3 The change of coordinate formula for the flow $\left(w_{\alpha j}^{i}(\mathbf{u}) u_{x}^{j}\right)_{x}$ into the flow $\left(w_{\alpha j}^{i}(\mathbf{q}) q_{x}^{j}\right)_{x}$ is

$$
\frac{\partial q^{k}}{\partial u^{h}} w_{i}^{h}(\mathbf{u}) \frac{\partial u^{i}}{\partial q^{j}}=w_{j}^{k}(\mathbf{q})
$$

We remain with the computational problem of expressing $\left(u^{i}\right)$ in terms of $\left(q^{k}\right)$. This would lead to complicated expressions involving roots, so we will write down the matrix $w_{\alpha k j}$ for the two flows $(a=1,2)$ using the coordinates $\left(u^{i}\right)$ as parameters for $\left(q^{k}\right)$. The two matrices $w_{\alpha k j}$ turn out to be skewsymmetric, so that the condition (14a) is satisfied. All coefficients $w_{\alpha k j}$ have denominators that contain factors of

$$
\Delta=\sqrt{\left|\operatorname{det}\left(g_{i j}\right)\right|}=\left(u^{1}-u^{2}\right)\left(u^{1}-u^{3}\right)\left(u^{2}-u^{3}\right) u^{5}
$$

if we introduce the notation $\tilde{w}_{\alpha k j}=\Delta w_{\alpha k j}$, then the only nonzero components of the two matrices $\tilde{w}_{\alpha k j}($ for $k<j$ ) are

$$
\begin{aligned}
& \tilde{w}_{113}=\frac{-\left(u^{2}+u^{3}-u^{1}-u^{6}\right)\left(u^{2}-u^{3}\right) u^{5}}{2} \\
& \tilde{w}_{114}=\left(u^{2}-u^{3}\right)\left(u^{5}\right)^{2} \\
& \tilde{w}_{125}=\frac{-\left(u^{2}+u^{3}-u^{1}-u^{6}\right)\left(u^{2}-u^{3}\right) u^{5}}{2} \\
& \tilde{w}_{126}=\left(u^{2}-u^{3}\right)\left(u^{5}\right)^{2} \\
& \tilde{w}_{134}=\left(u^{2}-u^{3}\right)\left(u^{5}\right)^{2} u^{1} \\
& \tilde{w}_{135}=\frac{-\left(4 u^{4} u^{5}+\left(u^{6}\right)^{2}-2 u^{6} u^{1}+\left(u^{1}\right)^{2}-\left(u^{2}\right)^{2}+2 u^{2} u^{3}-\left(u^{3}\right)^{2}\right)\left(u^{6}+u^{1}-u^{2}-u^{3}\right)\left(u^{2}-u^{3}\right)}{8} \\
& \tilde{w}_{145}=\frac{-\left(4 u^{4} u^{5}+\left(u^{6}\right)^{2}-4 u^{6} u^{1}-\left(u^{1}\right)^{2}+2 u^{1} u^{2}+2 u^{1} u^{3}-\left(u^{2}\right)^{2}+2 u^{2} u^{3}-\left(u^{3}\right)^{2}\right)\left(u^{2}-u^{3}\right) u^{5}}{4} \\
& \tilde{w}_{146}=\left(u^{2}-u^{3}\right)\left(u^{5}\right)^{2} u^{1} \\
& \tilde{w}_{156}=\frac{-\left(4 u^{4} u^{5}+\left(u^{6}\right)^{2}-2 u^{6} u^{1}+\left(u^{1}\right)^{2}-\left(u^{2}\right)^{2}+2 u^{2} u^{3}-\left(u^{3}\right)^{2}\right)\left(u^{6}+u^{1}-u^{2}-u^{3}\right)\left(u^{2}-u^{3}\right)}{8}
\end{aligned}
$$


and

$$
\begin{aligned}
& \tilde{w}_{213}=\frac{-\left(u^{6}-u^{1}+u^{2}-u^{3}\right)\left(u^{1}-u^{3}\right) u^{5}}{2} \\
& \tilde{w}_{214}=-\left(u^{1}-u^{3}\right)\left(u^{5}\right)^{2} \\
& \tilde{w}_{225}=\frac{-\left(u^{6}-u^{1}+u^{2}-u^{3}\right)\left(u^{1}-u^{3}\right) u^{5}}{2} \\
& \tilde{w}_{226}=-\left(u^{1}-u^{3}\right)\left(u^{5}\right)^{2} \\
& \tilde{w}_{234}=-\left(u^{1}-u^{3}\right)\left(u^{5}\right)^{2} u^{2} \\
& \tilde{w}_{235}=\frac{\left(4 u^{4} u^{5}+\left(u^{6}\right)^{2}-2 u^{6} u^{2}-\left(u^{1}\right)^{2}+2 u^{1} u^{3}+\left(u^{2}\right)^{2}-\left(u^{3}\right)^{2}\right)\left(u^{6}-u^{1}+u^{2}-u^{3}\right)\left(u^{1}-u^{3}\right)}{8} \\
& \tilde{w}_{245}=\frac{-\left(\left(u^{2}-u^{3}\right)^{2}+\left(u^{1}\right)^{2}-2\left(u^{2}+u^{3}\right) u^{1}-\left(u^{6}-4 u^{2}\right) u^{6}-4 u^{4} u^{5}\right)\left(u^{1}-u^{3}\right) u^{5}}{4} \\
& \tilde{w}_{246}=-\left(u^{1}-u^{3}\right)\left(u^{5}\right)^{2} u^{2} \\
& \tilde{w}_{256}=\frac{\left(4 u^{4} u^{5}+\left(u^{6}\right)^{2}-2 u^{6} u^{2}-\left(u^{1}\right)^{2}+2 u^{1} u^{3}+\left(u^{2}\right)^{2}-\left(u^{3}\right)^{2}\right)\left(u^{6}-u^{1}+u^{2}-u^{3}\right)\left(u^{1}-u^{3}\right)}{8}
\end{aligned}
$$

We end this paper by exhibiting the third-order Hamiltonian operator for the Oriented Associativity equation in hydrodynamic form.

Theorem 4 The Oriented Associativity equation in hydrodynamic form (7) admits the non-local third-order Dubrovin-Novikov Hamiltonian operator

$$
\begin{aligned}
B= & \partial_{x}\left(g^{i j} \partial_{x}+c_{k}^{i j} u_{x}^{k}+c^{1} w_{1 k}^{i} u_{x}^{k} \partial_{x}^{-1} w_{1 h}^{j} u_{x}^{h}\right. \\
& \left.+c^{2}\left(w_{1 k}^{i} u_{x}^{k} \partial_{x}^{-1} w_{2 h}^{j} u_{x}^{h}+w_{2 k}^{i} u_{x}^{k} \partial_{x}^{-1} w_{1 h}^{j} u_{x}^{h}\right)+c^{3} w_{2 k}^{i} u_{x}^{k} \partial_{x}^{-1} w_{2 h}^{j} u_{x}^{h}\right) \partial_{x}
\end{aligned}
$$

where $g^{i j}$ is the inverse of the Monge metric (24), $c_{k}^{i j}$ are defined through $g^{i j}$, $w_{1 j}^{i}$ and $w_{2 j}^{i}$ are the matrices of the two commuting flows that we found above and

$$
c^{1}=2, \quad c^{2}=1, \quad c^{3}=2 .
$$

Proof. It is only necessary to check the conditions

$$
\begin{aligned}
& c_{i j k, l}+g^{p q} c_{p j k} c_{q i l}+ \\
& c^{1} w_{1 j k} w_{1 i l}+c^{2}\left(w_{1 j k} w_{2 i l}+w_{1 i l} w_{2 j k}\right)+c^{3} w_{2 j k} w_{2 i l}=0, \\
& w_{a i j, s} \frac{\partial u^{s}}{\partial q^{l}}-g^{p q} c_{p i j} w_{a q l}=0, \quad a=1,2
\end{aligned}
$$

which yield the Hamiltonian property with the given values of the constants $c^{i}$. 


\section{Acknowledgments}

We thank Matteo Casati, Boris Dubrovin, Evgeny Ferapontov and Paolo Lorenzoni for stimulating discussions.

This work was partially supported by the grant of Presidium of RAS "Fundamental Problems of Nonlinear Dynamics" and by the RFBR grant 17-01-00366. RV acknowledges the support of Dipartimento di Matematica e Fisica "E. De Giorgi" of the Università del Salento, of Istituto Nazionale di Fisica Nucleare by by IS-CSN4 Mathematical Methods of Nonlinear Physics, of GNFM of Istituto Nazionale di Alta Matematica http://www . altamatematica.it.

The authors thank the Centro Internazionale per la Ricerca Matematica (Trento, Italy) for its hospitality in March 2018 within the framework of a 'research in pairs' program, where this paper was completed.

\section{References}

[1] A. Arsie, P. Lorenzoni, F-manifolds, multi-flat structures and Painlevé transcendents, arXiv: 1685937.

[2] M. Casati, E.V. Ferapontov, M.V. Pavlov and R.F. Vitolo, On a class of third-order non-local Hamiltonian operators, J. Geom. Phys. (2018), https://doi.org/10.1016/j.geomphys.2018.10.018, https://arxiv.org/abs/1805.00746.

[3] B.A. Dubrovin, Geometry of 2D topological field theories, Lecture Notes in Math. 1620, Springer-Verlag (1996) 120-348.

[4] B.A. Dubrovin and S.P. Novikov, Hamiltonian formalism of one-dimensional systems of hydrodynamic type and the Bogolyubov-Whitham averaging method, Soviet Math. Dokl., 27 (1983) 781-785.

[5] B.A. Dubrovin and S.P. Novikov, Poisson brackets of hydrodynamic type, Soviet Math. Dokl. 30 No. 3 (1984), 651-654.

[6] E.V. Ferapontov, Nonlocal Hamiltonian operators of hydrodynamic type: differential geometry and applications, Amer. Math. Soc. Transl. (2), 170 (1995) $33-58$.

[7] E.V. Ferapontov, Differential geometry of nonlocal Hamiltonian operators of hydrodynamic type, Func. Anal. Appl., 25 No. 3 (1991) 37-49. 
[8] E.V. Ferapontov, C.A.P. Galvao, O.I. Mokhov and Y. Nutku, Bi-Hamiltonian structure of equations of associativity in 2-d topological field theory, Comm. in Math. Phys., 186 (1997) 649-669.

[9] E.V. Ferapontov and O.I. Mokhov, On the Hamiltonian representation of the associativity equations. In: Algebraic aspects of integrable systems: In memory of Irene Dorfman. Eds. I.M. Gelfand, A.S. Fokas, Birkhäuser, Boston, 1996, 75-91.

[10] E.V. Ferapontov, M.V. Pavlov and R.F. Vitolo Projective-geometric aspects of homogeneous third-order hamiltonian operators. J. Geom. Phys., 85:16-28, 2014. DOI:10.1016/j.geomphys.2014.05.027.

[11] E.V. Ferapontov, M.V. Pavlov and R.F. Vitolo, Towards the classification of homogeneous third-order Hamiltonian operators. Int. Math. Res. Not., 22:6829-6855, 2016.

[12] E.V. Ferapontov, M.V. Pavlov and R.F. Vitolo, Systems of conservation laws with third-order Hamiltonian structures. Lett. Math. Phys., 108(6):15251550, 2018. https://arxiv.org/abs/1703.06173.

[13] C. Hertling and Y. Manin, Weak Frobenius manifolds, Internat. Math. Res. Notices 1999, no. 6, 277-286.

[14] P. Kersten, I. Krasil'shchik and A. Verbovetsky, Hamiltonian operators and $\ell^{*}$-coverings. J. Geom. Phys., 50:273-302, 2004.

[15] B. Konopelchenko and F. Magri, Coisotropic deformations of associative algebras and dispersionless integrable hierarchies. Comm. Math. Phys. 274 No. 3 (2007) 627-658.

[16] B.G. Konopelchenko and G. Ortenzi, Coisotropic deformations of algebraic varieties and integrable systems. J. Phys. A: Math. Theor. 42 No. 41 (2009) 415207, $18 \mathrm{pp}$.

[17] J. Krasil'shchik, A. Verbovetsky and R. Vitolo, The symbolic computation of integrability structures for partial differential equations. Texts and Monographs in Symbolic Computation. Springer, 2018. ISBN 978-3-319-71654-1; see http://gdeq.org/Symbolic_Book for downloading program files that are discussed in the book.

[18] P. Lorenzoni, M. Pedroni and A. Raimondo, F-manifolds and integrable systems of hydrodynamic type, Archivum Mathematicum 47 (2011), 163-180. 
[19] P. Lorenzoni, Darboux-Egorov System, Bi-flat F-Manifolds and Painlevé VI, IMRN 12 (2014), 3279-3302.

[20] F. Magri, A simple model of the integrable Hamiltonian system, J. Math. Phys., 19 No. 5 (1978) 1156-1162.

[21] O.I. Mokhov and E.V. Ferapontov, Non-local Hamiltonian operators of hydrodynamic type related to metrics of constant curvature, Russ. Math. Surv. no. 345 (1990), 218-219.

[22] M. Pavlov and A. Sergyeyev, Oriented associativity equations and symmetry consistent conjugate curvilinear coordinate nets, J. Geom. Phys. 85 (2014), $46-59$.

[23] M.V. Pavlov and R.F. Vitolo, On the bi-Hamiltonian geometry of WDVV equations, Lett. Math. Phys. 105, no. 8 (2015), 1135-1163.

[24] R. Vitolo, Computing with Hamiltonian Operators, https://arxiv.org/abs/1808.03902. 